\title{
Investigation of intensity laser effects on environment
}

\begin{abstract}
In this paper different types of weather conditions which effect laser beam quality are being study. Atmospheric conditions such as rain, snow, fog and dust are discussed. Atmospheric turbulence effect on size and intensity of laser beam is described and background optical power relations and received power by receiver are presented. Ground target reflection coefficient and related charts are presented.
\end{abstract}

Keywords: laser, atmosphere, scattering, turbulence
Volume 4 Issue 2 - 2020

\author{
Arash Rezaei,' Hamid Motavalibashi, ${ }^{2}$ Milad \\ Farasat $^{3}$ \\ 'Farhangian university of Isfahan, Isfahan, Iran \\ ${ }^{2}$ Iran aircraft Company Isfahan, Isfahan, Iran \\ ${ }^{3}$ Farhangian university of Isfahan, Isfahan, Iran
}

Correspondence: Arash Rezae, Farhangian university of Isfahan, Isfahan, Iran, Email arashrezaei585@gmail.com

Received: November 17, 2019 | Published: April 30, 2020

\section{Introduction}

In research, especially in the military field, extensive studies have been conducted on guided laser equipment. Use of laser-guided equipment began in America Air Force during the Vietnam War since the 1970s. The first advantage of this type of weapons was confirmed in this war proved in precision destroying targets with relatively small dimensions but with high importance. Today, laser weapons are used much wider for operations against enemy targets armor and protection. The biggest advantage of this type of weaponry is their high precision that can destroy the precise goals with the least amount of ammunition. Another advantage of using these weapons is the possibility of targeting a point precisely for consecutive times that is very efficient to eliminate armor targets. In recent years, military studies and the correct choice of materials for the manufacture of tracker systems pay special attention to laser detectors. Before transmitting a laser beam inside the environment, it is important to identify the effects of the environment on the beam. Molecules and particles such as dust, fog, smoke, steam, and aerosols, have significant effects on the dispersion, reflection and absorption of laser beams, which themselves contribute to the degradation, deviation, and reduction of laser beam coherence. In this paper, the effects of the environment on the laser beam and the ways to reduce these effects are examined.

\section{Discussion}

Atmospheric obscurants reduce the performance of sensors by reducing the signal radiation reaching the sensor because of reduced atmosphere transmittance in the sensor wave length response region, increasing noise at the sensor due to scattering of atmosphering radiation or system illuminator energy into the sensor and reducing the signal - to - noise ratio through turbulence induced wave - front degradation. The three curves indicate a tropical atmosphere with high water vapor content, a subarctic atmosphere, which has a low water vapor content, and a typical us or midlatitude atmosphere, which has a moderate contant. These curves illustrate the effect of water vapor contant on thermal transmittance. Extinction is defined as the reduction, or attenuation of radiation passing through atmosphere.
Extinction comprises two process: absorption of energy and scattering of energy. In absorption, a photon of radiation is absorbed by an atmosphere molecular or an aerosol particle. In scattering, the direction of the incident radiation is changed by collisions with atmospheric molecular or aerosol particle. Absorption usually dominates scattering at IR and $\mathrm{mmw}$ wave length. Scattering is the major factor in visible extinction but may also be important at IR wave length.

Scattering effectiveness is given by the scattering efficiency Q (n, r) which is ratio of the effective scattering cross of a particle of radius $r$ to its geometric cross section as: $:^{1,2}$

$$
Q(\lambda, r)=\frac{\dot{o}_{\mathrm{s}}}{\pi \mathrm{r}^{2}}=\frac{2}{\mathrm{r}^{2}} \int_{0}^{\gamma} \sigma_{\mathrm{s}}(\theta) \sin \theta \mathrm{d} \theta
$$

Where

$$
\begin{aligned}
& \mathrm{r}=\text { particle radius, } \mathrm{m} \\
& \begin{aligned}
\sigma & =\text { angular scattering cross section, } \mathrm{m} / \mathrm{sr} \\
\theta & =\text { scattering angle, } \mathrm{rad}
\end{aligned}
\end{aligned}
$$

If the particle size is much smaller than the radiation wave length, Rayliegh scattering results, and scattering efficiency simplifies to the expression: ${ }^{1}$

$$
Q(\lambda, r)=\frac{8}{3}(2 \pi)^{4} \frac{r^{4}\left[n(\lambda)^{2}-1\right]^{2}}{\lambda^{4}\left[n(\lambda)^{2}+2\right]^{2}}
$$

Where

$\mathrm{n}(\lambda)=$ real part of index of refraction, $\mathrm{r}=$ particle radius, $m$.

Particle size for several common obscurants are given in tables 1 and 2.If the particle size is much larger than the radiation wave length, scattering efficiency calculated by geometrical scattering. According to the table 2 , types of atmospheric particles (steam, aerosols, rain, snow ,...) have different scattering relations. 


\section{Steam}

The following equation shows the atmosphere transmittance coefficient for steam and molecular particles. ${ }^{1}$

$$
\mathrm{T}_{\mathrm{m}}(\ddot{\mathrm{e}})=\mathrm{e}^{-\tilde{\mathrm{O}}_{\mathrm{m}}(\ddot{\mathrm{e}}) \mathrm{R}}
$$

Where

$\mathrm{T}_{\mathrm{m}}=$ atmosphere transmittance coefficient for steam and molecular

$\tilde{\mathrm{O}}_{\mathrm{m}}=$ steam and molecular attenuation coefficient.

$\mathrm{R}=$ Path length

The average value of $\gamma$ for low humidity (lower water vaper than $3.5 \mathrm{~g} / \mathrm{m}^{3}$ ) in visible spectrum is between 0.4 and 0.7 and for high humidity (water vaper more than $14 \mathrm{~g} / \mathrm{m}^{3}$ ) is about 0.02 . In the near - infrared range (between 0.7 and 1.1) for low humidity the average value of $\gamma$ is about 0.02 and for high humidity is about 0.03 . The water vapor atmosphere transmittance coefficient within 3 to $5 \mu \mathrm{m}$ is specifid in table 3 and within 8 to $12 \mu \mathrm{m}$ in Table 4 . Also according to Table 5, the attenuation coefficient for 10.591 wave length and $\mathrm{mmw}$ in different humidities

Aerosol (fog, cloud, dust)

The equation below shows the atmosphere transmittance coefficient for aerosols: ${ }^{1}$

$$
\mathrm{T}_{\mathrm{a}}(\ddot{\mathrm{e}})=\mathrm{e}^{-\tilde{\mathrm{O}}_{\mathrm{a}}(\ddot{\mathrm{e}}) \mathrm{R}}
$$

where $\mathrm{T}_{\mathrm{a}}$ atmosphere transmittance coefficient

$\tilde{\mathrm{O}}_{\mathrm{a}}$ reduce coefficient of aerosols and $\mathrm{R}=$ path length. The average of $\mathrm{Y}_{\mathrm{a}}$ in visible spectrum (between 0.4 to 0.7 ) is:

$$
\tilde{\mathrm{O}}_{\mathrm{a}}(0.4-0.7 \mathrm{ì} \mathrm{m})=3.912 / \mathrm{V}
$$

Where $\mathrm{V}$ is visible distance in $\mathrm{km}$

Visible distance is defined as the distance which you can diagnose an object properly by the contrast of 1 against background by the contrast of $0.02 .{ }^{1}$ In Table 6 visibility is provided for different regions. In IR area for the yag laser: two values will be obtained for aerosols attenuation coefficient one for visible distance more than $0.6 \mathrm{~km}$ which is equal to: ${ }^{1}$

$$
\tilde{\mathrm{O}}_{\mathrm{a} 1}(1.06 \mathrm{ì} \mathrm{m})=10^{[-0.136+1.16 \log (3.912 / \mathrm{V})]}
$$

And for a visible distance less than $0.6 \mathrm{~km}$ and equal to $0.6 \mathrm{~km}$ :

$$
\tilde{\mathrm{O}}_{\mathrm{a} 2}(1.06 \grave{\mathrm{m}})=3.912 / \mathrm{V}
$$

In near-IR range (between 0.7 to 1.1 ) the average value of $\mathrm{Y}_{\mathrm{a}}$ is equal to:

$$
\tilde{\mathrm{O}}_{\mathrm{a}}(0.7-1.1 \mathrm{ì} \mathrm{m})=0.6(3.912 / \mathrm{V})
$$

Table7 shows the attenuation coefficient for other wavelength (Table 7).

Radiation fog forms when the weather cools down until the dew point and advection fog forms when vertical air mixture with different temperatures manufactures until the dew point. In these two types the size of fog particle are different.

\section{Rain:}

The following equation defines the atmosphere transmittance coefficient for the precipitation in the air.

$$
\mathrm{T}_{\mathrm{p}}(\ddot{\mathrm{e}})=\mathrm{e}^{-\tilde{\mathrm{O}}_{\mathrm{p}}(\ddot{\mathrm{e}}) \mathrm{R}}
$$

Where

$. \mathrm{T}_{\mathrm{p}}=$ the atmosphere transmittance coefficient for the precipitation in the air

$$
\text { . } \tilde{\mathrm{O}}_{\mathrm{p}}=\text { the precipitation attenuation coefficient }
$$

$\mathrm{R}=$ path length

The average value of $\tilde{O}_{p}$ (in visible spectrum range to thermal wavelength) determines based on the amount of rainfall for three different types of rainfall.

For the drizzle we have: ${ }^{1}$

$$
\tilde{\mathrm{O}}_{\text {prd }}(\text { Visible }- \text { Thermal })=0.51 r^{0.63}
$$

For the widespread we have:

$$
\tilde{\mathrm{O}}_{\text {prw }}(\text { Visible }- \text { Thermal })=0.36 r^{0.63}
$$

And for the thunderstorm we have:

$$
\tilde{O}_{\text {prt }}(\text { Visible }- \text { Thermal })=0.16 r^{0.63}
$$

Where $\mathrm{r}=$ amount of rainfall ( $\mathrm{mm}$ per hour $(\mathrm{mmph}))$

\section{Snowfall:}

The atmosphere transmittance coefficient equation for both rainfall and snowfall are the same. The only difference is the attenuation coefficient. The snowfall attenuation coefficient depends on visible distance and equals to: ${ }^{1}$

$$
\tilde{\mathrm{O}}_{\mathrm{ps}}(\text { Visible }- \text { Thermal })=3.912 / \mathrm{V}
$$

Dust:

The following equation defines the atmosphere transmittance coefficient for dust in the air. ${ }^{1}$

$$
\mathrm{T}_{\mathrm{d}}(\ddot{\mathrm{e}})=\mathrm{e}^{-\mathrm{a}} \mathrm{d}(\ddot{\mathrm{e}}) \mathrm{Cl}
$$

Where

. $\mathrm{T}_{\mathrm{d}}=$ Atmosphere transmittance coefficient for dust in the air

. ${ }_{\mathrm{d}}=$ Attenuation coefficient of dust in the air

And $\mathrm{cl}=$ Path length density by $\mathrm{g} / \mathrm{m}^{2}$

$\mathrm{Cl}$ achieves from multiplication of upload mass by path length $\mathrm{R}$ generally for the a we could write. ${ }^{1}$

$$
\dot{a}_{\mathrm{d}}(\ddot{\mathrm{e}})=<<\frac{Q \sigma}{M}>>
$$

Where:

$\sigma=$ Cross-sectional area of particle

$\mathrm{Q}=$ The dispersion coefficient

$\mathrm{M}=$ Mass of the particle 
The internal bracket is identifier of solid angle average and the external bracket is the identifier of mass distribution average of the particle. For the cl we have:

$$
C l=\int_{n}^{n_{2}} C(r) d l
$$

Where

$$
\begin{aligned}
& C(r)=\text { density at } \mathrm{r} \\
& . d l=\text { longitudinal element of the doped area } \\
& r_{2}-r_{1}=\text { length of the doped area }
\end{aligned}
$$

The Table 9 shows the dust attenuation coefficient for different wavelength

\section{Smoke:}

The following equation defines the atmosphere transmittance coefficient for smoke in the air: ${ }^{1}$

$$
\mathrm{T}_{\mathrm{s}}(\ddot{\mathrm{e}})=\mathrm{e}^{-\dot{a}_{\mathrm{s}}(\ddot{\mathrm{e}}) \mathrm{Cl}}
$$

Where

$\mathrm{T}_{\mathrm{s}}=$ atmosphere transmittance coefficient for smoke in the air

. $\mathrm{a}_{\mathrm{s}}=$ attenuation coefficient of smoke in the air,g/m²

And $\mathrm{cl}=$ path length of density, $\mathrm{g} / \mathrm{m}^{2}$

$\mathrm{Cl}$ also defines according to the equation (17)

According to the equation 17 we could calculate the amount of $\mathrm{cl}$ so that 1 is the length of infected area then due to the equation 16 we could calculate the atmosphere transmittance coefficient for smoke for the all of atmosphere transmittance coefficient in the absence of precipitation we have:

$$
T(\ddot{\mathrm{e}})=\mathrm{T}_{m}(\ddot{\mathrm{e}}) \mathrm{T}_{a}(\ddot{\mathrm{e}}) \mathrm{T}_{s}(\ddot{\mathrm{e}}) \mathrm{T}_{d}(\ddot{\mathrm{e}})
$$

And in presence of snowfall or rainfall we have:

$$
T(\ddot{\mathrm{e}})=\mathrm{T}_{m}(\ddot{\mathrm{e}}) \mathrm{T}_{p}(\ddot{\mathrm{e}}) \mathrm{T}_{s}(\ddot{\mathrm{e}}) \mathrm{T}_{d}(\ddot{\mathrm{e}})
$$

\section{Light turbulence}

The atmospheric turbulence reduces by wavelength enhancement. Atmospheric turbulence causes beam extension beam divagation flashing and fluctuation in the brightness of the beam. ${ }^{4}$ These effect will be describe by radius of beam displacement of the center of beam compatibility or confliction of radiation of the beam. The scintillation effect causes the reduction of pendulous power average at the receiver aperture. Movement of picture or the blur of the caused turbulence describe by optic function (coherence length) and also wave front tilt. The atmospheric turbulence could be considered as a compound of cell with different size and refractive index. These cells move within the beam and cause the effect which is explained at the above. Assuming still and freezed atmosphere the speed and direction of this uniform movement determines by the wind average speed. Based on the size of dominant cell and beam diameter the turbulence cells cause the beam scattering in different direction. When size of the cell is smaller than the beam diameter refraction and diffraction happens. The beam radiation figure turns into a small ray and the dark area results of interference of wave front refraction and diffraction (flicker). Based on the turbulence power ratio each one of the two cases of the above may be observed singly or together. Strehl is the ratio of the average of radiation on the axis with turbulence to the average of radiation on the axis without turbulence .so that the ratio of the beam diameter with turbulence to the beam without turbulence is equal to:

For the long term turbulence cases we have: ${ }^{1}$

$$
S_{l}=\left[1+\left(D / r_{0}\right)^{2}\right]^{-1}
$$

And for the short term turbulence if $\left(\mathrm{D} / \mathrm{r}_{0}\right) \leq 3$ :

$$
S_{s 1}=\left[1+0.182\left(D / r_{0}\right)^{2}\right]^{-1}
$$

And if $\left(D / r_{0}\right)>3$ :

$$
S_{s 2}=\left[1+\left(D / r_{0}\right)^{2}-1.18\left(D / r_{0}\right)^{5 / 3}\right]^{-1}
$$

Where

$\mathrm{D}=$ effective diameter of the laser aperture

. $S_{l}=$ ratio of the long term strehl

. $S_{s}=$ ratio of the short term strehl

And $r_{0}=$ coherence length

If the turbulence is uniform we have:

$$
r_{0}=0.3325\left(10^{-6} \lambda\right)^{6 / 5}\left(10^{3} C_{n}^{2} R\right)^{-3 / 5}
$$

Where $C_{n}^{2}=$ constant of the refraction index by $m^{-2 / 3}$

The amount of $C_{n}^{2}$ changes between $10^{-14}$ for the weak turbulence $6 * 10^{-14}$ for the medium turbulence and $6 * 10^{-13}$ for hard turbulence

For the non-uniform turbulence this effect is strong the flicker effect due to atmosphere is estimated by the following equation

$$
\mathrm{o}_{I}^{2}=1.24 \mathrm{C}_{n}{ }^{2}(2 \pi / \lambda)^{7 / 6}\left(10^{3} R\right)^{11 / 6}
$$

In the spread range or hard turbulence the amount of $\dot{o}_{I}^{2}$ wont be more than 0.5 sigma is by $\mathrm{w}$ for the consubstantial turbulence the amount of $o_{x}^{2}$ variance of the movement of the center of the picture is equal to:

$$
\dot{o}_{x}^{2}=1.093 \mathrm{C}_{n}^{2} F^{2} D^{-1 / 3} 10^{3} R
$$

Where $\mathrm{f}=$ focal length of the receiver

The reflection coefficient of ground targets 
At first reflection coefficient is reviewed for very important ground targets. Generally natural targets are divided to 5 total categories which three categories of water cloud and snow due to close nature are mentioned in one. ${ }^{5,6}$

i. Agricultural land trees bushes and meadows

ii. Ground

iii. Rocks

iv. Water cloud and snow

\section{v. Metals}

\section{Received power}

To determination of the most range of object location and tracing the lighten object by the laser pulse first of all it's necessary to find the light level in optical receiver sensitive position. It's specified as well that the angel measurement error is highly dependent to noise signal ratio in output receiver ring. In more analysis according to Table (1-7) geometric characteristic of the laser, the lighten object and the optical receiver are in use to specify the optical power level at the optical receiver input (Table 1$)^{7}$

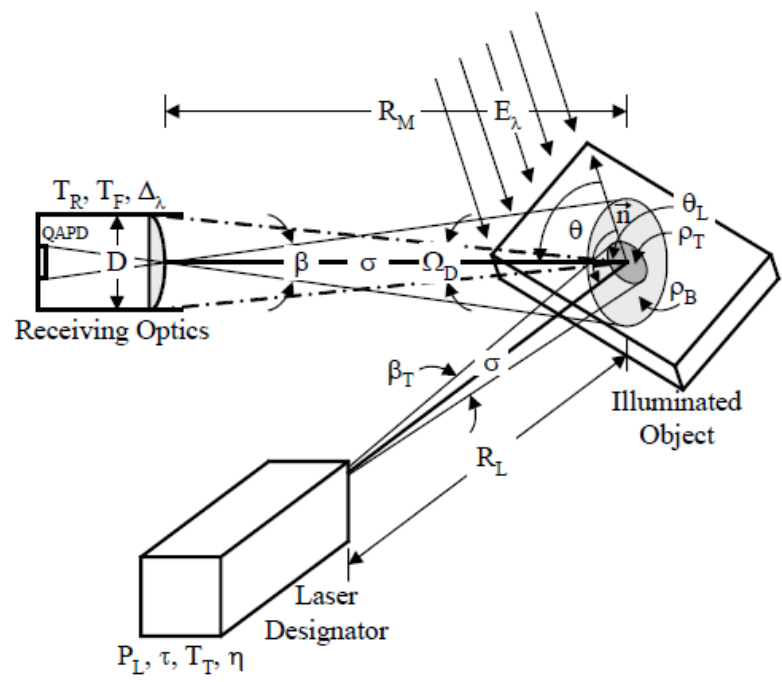

Table I The effect of the particles on the order dispersion wave length'

\begin{tabular}{lll}
\hline Effect & Distribution type & Radius of particle \\
\hline Symmetric distribution & Rayleigh scattering & less than $\lambda / 10$ \\
Most distribution & Mie scattering & more than $\lambda / 10$ \\
Most scatting forward & Mie scattering & about $\lambda / 4$ \\
All scatting forward & Mie scattering & more than $\lambda$ \\
Refraction, reflection, diffraction & Light scatting geometry & more than $10 \lambda$ \\
\hline
\end{tabular}

Table 2 Particle size distribution and the effect of atmospheric turbulence

\begin{tabular}{|c|c|c|c|c|}
\hline mm waves & Ir waves & vision waves & Diameter of particle $(\mu \mathrm{m})$ & Particle size \\
\hline Rayleigh scattering & Rayleigh scattering & Rayleigh scattering & $10^{-4}$ & $\begin{array}{l}\text { Atmosphere } \\
\text { Molecule }\end{array}$ \\
\hline Rayleigh scattering & Rayleigh scattering & Rayleigh and Mie scattering & $10^{-2}$ to $10^{-1}$ & Haze \\
\hline Rayleigh scattering & Mie scattering & $\begin{array}{l}\text { Mie and geometric } \\
\text { scattering }\end{array}$ & 0.5 to 100 & Fog \\
\hline Rayleigh scattering & Mie scattering & $\begin{array}{l}\text { Mie and geometric } \\
\text { scattering }\end{array}$ & 2 to 200 & Cloud \\
\hline Mie scattering & $\begin{array}{l}\text { Geometric } \\
\text { scattering }\end{array}$ & Geometric scattering & $10^{2}$ to $10^{4}$ & Rain \\
\hline $\begin{array}{l}\text { Mie and geometric } \\
\text { scattering }\end{array}$ & $\begin{array}{l}\text { Geometric } \\
\text { scattering }\end{array}$ & Geometric scattering & $5 \times 10^{3}$ to $\times 510^{5}$ & Show \\
\hline Rayleigh scattering & Mie scattering & $\begin{array}{l}\text { Mie and geometric } \\
\text { scattering }\end{array}$ & 1 & Smoke \\
\hline Rayleigh scattering & Mie scattering & $\begin{array}{l}\text { Mie and geometric } \\
\text { scattering }\end{array}$ & I to 100 & Dust \\
\hline
\end{tabular}


Table 3 Transmission coefficient for water vapor in the atmosphere of moisture and different distances ranging from 3-5 $\mu m$ ।

\begin{tabular}{|c|c|c|c|c|c|c|c|}
\hline \multicolumn{8}{|c|}{ Transmission coefficient for water vapor in the atmosphere of moisture $T_{m}(\lambda)$} \\
\hline \multicolumn{6}{|c|}{ Along the way in terms $\mathrm{km}$} & \multirow{2}{*}{$\begin{array}{l}\text { Temperature } \\
\left({ }^{\circ} C\right)\end{array}$} & \multirow[t]{2}{*}{ The moisture content } \\
\hline 15 & 10 & 7 & 5 & 3 & I & & \\
\hline 0.47 & 0.53 & 0.58 & 0.62 & 0.68 & 0.77 & 0 & \\
\hline 0.42 & 0.48 & 0.53 & 0.58 & 0.61 & 0.74 & 10 & 10 \\
\hline 0.38 & 0.44 & 0.49 & 0.53 & 0.6 & 0.71 & 20 & \\
\hline 0.33 & 0.39 & 0.44 & 0.48 & 0.55 & 0.67 & 30 & \\
\hline 0.35 & 0.41 & 0.47 & 0.51 & 0.58 & 0.7 & 0 & \\
\hline 0.3 & 0.36 & 0.41 & 46 & 0.53 & 0.66 & 10 & 40 \\
\hline 0.24 & 0.3 & 0.35 & 0.4 & 0.47 & 0.61 & 20 & \\
\hline 0.19 & 0.25 & 0.3 & 0.35 & 0.42 & 0.56 & 30 & \\
\hline 0.3 & 0.36 & 0.41 & 0.46 & 0.53 & 0.66 & 0 & \\
\hline 0.24 & 0.3 & 0.35 & 0.4 & 0.47 & 0.61 & 10 & 70 \\
\hline 0.18 & 0.24 & 0.29 & 0.34 & 0.41 & 0.56 & 20 & \\
\hline 0.13 & 0.18 & 0.23 & 0.28 & 0.36 & 0.5 & 30 & \\
\hline 0.27 & 0.33 & 0.39 & 0.44 & 0.51 & 0.64 & 0 & \\
\hline 0.21 & 0.27 & 0.33 & 0.37 & 0.45 & 0.59 & 10 & \\
\hline 0.15 & 0.21 & 0.26 & 0.31 & 0.39 & 0.53 & 20 & 90 \\
\hline 0.1 & 0.15 & 0.2 & 0.25 & 0.33 & 0.48 & 30 & \\
\hline
\end{tabular}

Table 4 Transmission coefficient for water vapor in the atmosphere of moisture and different distances ranging from 8-I $2 \mu m$ ।

\begin{tabular}{|c|c|c|c|c|c|c|c|}
\hline \multicolumn{6}{|c|}{ Along the way in terms $\mathrm{km}$} & \multirow{2}{*}{$\begin{array}{l}\text { Temperature } \\
\left({ }^{o} \mathrm{C}\right)\end{array}$} & \multirow[t]{2}{*}{ The moisture content } \\
\hline 15 & 10 & 7 & 5 & 3 & I & & \\
\hline 0.86 & 0.89 & 0.91 & 0.93 & 0.95 & 0.97 & 0 & \\
\hline 0.82 & 0.86 & 0.89 & 0.91 & 0.93 & 0.97 & 10 & 10 \\
\hline 0.76 & 0.81 & 0.85 & 0.87 & 0.91 & 0.95 & 20 & \\
\hline 0.65 & 0.72 & 0.78 & 0.82 & 0.87 & 0.94 & 30 & \\
\hline 0.72 & 0.78 & 0.82 & 0.86 & 0.89 & 0.95 & 0 & \\
\hline 0.55 & 0.65 & 0.72 & 0.77 & 0.84 & 0.92 & 10 & 40 \\
\hline 0.31 & 0.43 & 0.54 & 0.62 & 0.73 & 0.87 & 20 & \\
\hline 0.09 & 0.18 & 0.28 & 0.39 & 0.54 & 0.78 & 30 & \\
\hline 0.56 & 0.66 & 0.73 & 0.78 & 0.84 & 0.93 & 0 & \\
\hline 0.3 & 0.42 & 0.53 & 0.62 & 0.73 & 0.87 & 10 & 70 \\
\hline 0.07 & 0.15 & 0.26 & 0.36 & 0.52 & 0.77 & 20 & \\
\hline 0 & 0.02 & 0.05 & 0.11 & 0.25 & 0.59 & 30 & \\
\hline 0.46 & 0.57 & 0.66 & 0.72 & 0.8 & 0.91 & 0 & \\
\hline 0.18 & 0.3 & 0.41 & 0.51 & 0.64 & 0.83 & 10 & \\
\hline 0.02 & 0.06 & 0.13 & 0.023 & 0.39 & 0.69 & 20 & 90 \\
\hline 0 & 0 & 0.01 & 0.04 & 0.12 & 0.46 & 30 & \\
\hline
\end{tabular}


Table 5 Molecules attenuation coefficient for wavelength 10.59I $\mu m$ and millimeter waves at different Humidities'

\begin{tabular}{llcc}
\hline Absolute humidity in terms of $\mathbf{g} / \mathbf{m}^{3}$ & \multicolumn{4}{l}{ Attenuation coefficient $\Upsilon_{m}$ In terms of $K_{m}-^{\prime}$} \\
\hline & $10.591 \mu m$ & $35 \mathrm{GHz}$ & $94 \mathrm{GHz}$ \\
1 & 0.083 & 0.018 & 0.025 \\
3 & 0.091 & 0.021 & 0.043 \\
5 & 0.109 & 0.024 & 0.067 \\
10 & 0.185 & 0.032 & 0.108 \\
15 & 0.311 & 0.041 & 0.154 \\
20 & 0.383 & 0.049 & 0.201 \\
\hline
\end{tabular}

Aerosol (fog, cloud, dust)

Table 6 Visible distances for different regions ${ }^{3}$

\begin{tabular}{ll}
\hline 0 to 50 meter & Dense dust \\
50 to 200 meter & Thick dust \\
200 to 500 meter & The average dust \\
500 to 1000 meter & Dust weak \\
1000 to 2000 meter & Low dust \\
2000 to 4000 meter & Fog \\
4000 to 10000 meter & May the poor \\
10000 to 20000 meter & clean Air \\
20000 to 50000 meter & Very clean air \\
more than 50000 meter & Ultra-clean air \\
\hline
\end{tabular}

Table 7 Attenuation coefficient of suspended particles in the air for wavelength $10.591 \mu \mathrm{m}, 8-12 \mu \mathrm{m}, 3-5 \mu \mathrm{m}$ ।

\begin{tabular}{|c|c|c|c|}
\hline \multirow[t]{2}{*}{ Particle size } & \multicolumn{3}{|c|}{ Attenuation coefficient $\Upsilon_{a}$ in terms of $K_{m}-^{\prime}$} \\
\hline & I0.59I $\mu m$ & $8-12 \mu m$ & 3-5 $\mu \mathrm{m}$ \\
\hline \multicolumn{4}{|l|}{ May city } \\
\hline Visibility to $2 \mathrm{~km}$ & 0.16 & 0.18 & 0.29 \\
\hline Visibility to $5 \mathrm{~km}$ & 0.06 & 0.07 & 0.11 \\
\hline Visibility to $10 \mathrm{~km}$ & 0.03 & 0.04 & 0.6 \\
\hline Visibility to 15 km & 0.02 & 0.02 & 0.04 \\
\hline \multicolumn{4}{|l|}{ May incident } \\
\hline Visibility to $0.5 \mathrm{~km}$ & 1.7 & 2.4 & 10.1 \\
\hline Visibility to I km & 0.9 & 1.2 & 5.1 \\
\hline \multicolumn{4}{|l|}{ May rose } \\
\hline Visibility to $0.5 \mathrm{~km}$ & 8.9 & 9 & 8.4 \\
\hline Visibility to I km & 4.5 & 4.5 & 4.2 \\
\hline
\end{tabular}


Table 8 Precipitation

\begin{tabular}{ll}
\hline Rainfall intensity & Annual rate \\
\hline Heavy & More than $7.7 \mathrm{~mm} / \mathrm{h}$ \\
Average & 2.5 to $7.7 \mathrm{~mm} / \mathrm{h}$ \\
Light & Less than $2.5 \mathrm{~mm} / \mathrm{h}$ \\
\hline
\end{tabular}

Table 9 Dust in the air attenuation coefficient for different wavelengths ${ }^{\prime}$

\begin{tabular}{ll}
\hline Rainfall intensity & Annual rate \\
\hline Heavy & More than $7.7 \mathrm{~mm} / \mathrm{h}$ \\
Average & 2.5 to $7.7 \mathrm{~mm} / \mathrm{h}$ \\
Light & Less than $2.5 \mathrm{~mm} / \mathrm{h}$ \\
\hline
\end{tabular}

Table I 0 Mass loading of dust visible for different distances ${ }^{\prime}$

\begin{tabular}{ll}
\hline Visible distance, $\mathrm{Km}$ & Mass loading, $\mathbf{g} / \mathrm{m}^{3}$ \\
\hline 0.2 & $1.1 \times 10^{-1}$ \\
0.47 & $6.9 \times 10^{-2}$ \\
\hline 1 & $2.1 \times 10^{-2}$ \\
3.2 & $5.2 \times 10^{-3}$ \\
\hline
\end{tabular}

Table I I Smoke attenuation coefficient obtained from a variety of sources, to different wavelengths"

\begin{tabular}{|c|c|c|c|c|c|c|c|}
\hline \multirow{3}{*}{ Smoke sources } & \multicolumn{7}{|c|}{ Attenuation coefficient smoke } \\
\hline & \multicolumn{7}{|c|}{ wavelength in terms of micrometer } \\
\hline & $0.4-0.7$ & $0.7-1.2$ & 1.06 & $3-5$ & 8-12 & 10.6 & $35.94 \mathrm{GHz}$ \\
\hline \multicolumn{8}{|c|}{ Fuel evaporates into mechanical } \\
\hline & 6.58 & 4.59 & 3.48 & 0.25 & 0.02 & 0.02 & 0.001 \\
\hline \multicolumn{8}{|c|}{ Spray fuel into diesel engines } \\
\hline & 5.65 & 4.08 & 3.25 & 0.25 & 0.03 & 0.03 & 0.001 \\
\hline \multicolumn{8}{|c|}{ Burning phosphorus } \\
\hline & 4.05 & 1.77 & 1.37 & 0.29 & 0.83 & 0.38 & 0.001 \\
\hline \multicolumn{8}{|c|}{ Burning zinc compounds } \\
\hline & 3.66 & 2.67 & 2.28 & 10.19 & 0.04 & 0.03 & 0.001 \\
\hline Coal & 6 & 3.5 & 2 & 0.23 & 0.05 & 0.06 & 0.001 \\
\hline
\end{tabular}

In analysis, in order to generalize, it's assumed that laser and optical receiver are placed in different places.

Analysis of the power level of the background and reflected optical signal of laser behaives according to the main known equation which is radioscopy. For the background and object it's assumed that scattered reflections are reflected of the Lamberty surfaces. Also it's assumed that all of the laser beam is on the object which is lighten by laser.

\section{Background power}

Background optical power which receives from a optical receiver sensitive to location at entrance is equal to: ${ }^{7}$

$$
P_{B}=L_{\lambda} G T_{R} T_{F} T_{a t}
$$

Where

. $L_{\lambda}=$ son radiance spectrum

$\mathrm{G}=$ geometric factor

. $T_{R}=$ transference coefficient of optical receiver

. $T_{F}=$ transference coefficient of optical filter
And $T_{a t}=$ atmosphere transference coefficient

$\mathrm{G}$ the geometric factor is obtained from two small area radiative exchange factor and equals to:

$$
A_{D} \operatorname{COS} \theta \cdot A_{R} \operatorname{COS} \theta_{p o}
$$

Where

. $A_{D}=$ Quad detector footprint area in background

. $A_{R} \operatorname{COS} \theta_{p o}=$ The effective area of the photoreceptor

$\theta=$ The angle between the vector perpendicular to the surface of the object and filed lines between the object and the receiver

$\theta_{p o}=$ The angle between the vector perpendicular to the surface receptor filed with the line between the object and the receiver

. $R_{M}=$ The distance between the object and the photoreceptor

In cases where tracking and positioning is good, The photoreceptor is always face to the object so $\theta_{p o}=0$

For the whole radiance of the $\operatorname{sun} L_{\lambda}$ which caused by a diffuse reflector we have: 


$$
T_{a t}=e^{-\mathrm{Y} R M}
$$

Where

$E_{\lambda}=$ the whole of the sun .

And. $\rho_{B}=$ background reflect

The amount of $E_{\lambda}$ per wavelength can be obtained from standard charts

. $T_{a t}$ atmospheric transfer coefficient is obtained from the following relationship comes to

$$
T_{a t}=e^{-\mathrm{Y} R_{M}}
$$

Where

$\Upsilon=$ Atmospheric extinction coefficient.

The back ground power that obtained from equation(25),when combined with equations (26) and (27) has to be obtained as follows.

Where

$\Delta_{\lambda}=$ The whole bandwidth Optical Filter

. $\beta=$ photoreceptor visibility range

$D_{p o}=$ Diameter optical receiver

\section{Signal power}

P_S the optical signal received by the laser radiation reflected from the object is lightened with a laser when the laser beam crosssectional area of the object is smaller than, equals to:

$$
P_{S}=L_{T} A_{T} \Omega_{D} T_{R} T_{F} e^{-\mathrm{Y} R_{M}} \operatorname{COS} \theta
$$

Where

$\ldots L_{T}=$ Spectrum reflected from the object

. $A_{T}=$ The area of the laser spot on the object

And $\Omega_{D}$ in accordance with Figure 3-7 angle created by the opening of an optical receiver

. $L_{T}$ spectrum is ${ }^{7-9}$

$$
L_{T}=\frac{4 P_{L} T_{T} \eta \rho_{T} e^{-\mathrm{Y} R_{L}} \operatorname{COS} \theta_{L}}{\pi^{2} \beta_{T}^{2} R_{L}^{2}}
$$

Where

. $P_{L}=$ peak power laser

. $T_{T}=$ transmission coefficient of light

$\eta=$ efficiency of collection optical transmission

$\rho_{T}=$ target reflection coefficient

$\theta_{T}=$ angle between the vector perpendicular to the surface with a laser beam

. $\beta_{T}=$ divergence angle of the laser beam

and $R_{L}=$ distance between the laser and the object
$A_{T}$ area of the laser spot on the object of value follow below

$$
A_{T}=\frac{\pi R_{L}^{2} \beta_{T}^{2}}{4 \operatorname{CoS} \theta_{L}}
$$

And for $\Omega_{D}$ angle we have:

$$
\Omega_{D} \approx \frac{\pi D_{p o}{ }^{2}}{4 R_{M}^{2}}
$$

By combining equations (31) to (33) with equation (30), we finally have for the $P_{S}$

$$
P_{S}=\frac{D_{p o}^{2}}{4 R_{M}^{2}} P_{L} \rho_{T} T_{T} \mathrm{c} T_{R} T_{F} e^{-\mathrm{Y}\left(R_{L}+R_{M}\right)} \operatorname{COS} \theta
$$

\section{Conclusion}

According to the formula of laser attenuation by atmospheric conditions such as temperature, humidity, dust, rain, snow polished and smoke and ... which are dependent to the laser wavelength and the distance and the light intensity in order to minimize the laser must:

1. Reduse the target distance

2. Increase the incident laser beam intensity

3. Increaase the Selective laser wavelength because the more higher frequency, the more attenuation efficiency and Conversely is the same situation

4. The purpose of reflecting surface so that the reflection coefficient is higher, For example, hitting a building is better that the laser to hit on smooth surfaces such as metallic windows.

\section{Acknowledgments}

None.

\section{Conflicts of interest}

Author declares that there are no conflicts of interest.

\section{Funding}

None.

\section{References}

1. Department of defense handbook Washington DC. Quantitative Description of Obscuration Factors for Electro-Optical and Millimeter Wave System. DOD HDBK-178. 1986.

2. M Pendley. Air Warfare Battlelab Initiative for Stabilized Portable Optical Target Tracking Receiver. International Command and Control Reserch and Technology Symposium the Future of C2, $10^{\text {th }}$ ed, 2004

3. Henrik Andersson. Position Sensitive Detectors-Device Technology and Application in Spectroscopy. Mid Sweden University Doctoral Thesis 48, 2008;ISBN 978-91-85317-91-2,5-25.

4. Gerald C Holst, CCD Arrays. Cameras and displays. $2^{\text {nd }}$ ed, SPIE Optical Engineering Press, Washington USA, 1998.

5. KC Bahuguna, Prabhat Sharma, NS Vasan, et al. Laser Range Sensors. Defence Science Journal. 2007;57(6):881-890. 
6. Department of defense handbook, range laser safety, mil-hdbk-828B, $2^{\text {nd }}$ ed. 2011. p. 10-35.

7. Zarko P, Barbaric, Lazo M, et al. Optimization of Optical Receiver Parameters for Pulsed Laser Tracking Systems. IEEE Transactions on instrumentation and measurement. 2009;58(3):9-279.
8. Gerald C, Holst. Electro-optical imaging system performance, $12^{\text {th }} \mathrm{ed}$, SPIE Optical Engineering Press, Washington USA, 2000.

9. Hiroaki Ando, Hiroshi Kanbe, Tatsuya Kimura. Characteristics of Germanium Avalanche Photodiodes in the Wavelength Region of 1-1.6 $\mu \mathrm{m}$. IEEE Journal of Quantum Electronics. 1978;14(11):804-810. 